\title{
Ulnar Neuropathy Among Active Workers Based Upon Hand Diagram Ratings
}

\author{
Robert A. Werner, MD, Alfred Franzblau, MD, Bradley Evanoff, MD, Sheryl Ulin, PhD
}

\begin{abstract}
Background: Limited studies have estimated the prevalence of ulnar neuropathy (UN) in the workplace. Hand diagrams have been demonstrated to have a good sensitivity and specificity when attempting to identify patients with UN.

Objective: To determine the prevalence and associated risk factors for UN among active workers based on results of a hand diagram, and to determine the reliability of hand diagram scoring.

Design: Cross-sectional study.

Setting: Seven different industrial and clerical work sites.

Methods: A total of 501 active workers were screened. Subjects completed a hand diagram and the Job Content Questionnaire, and had ergonomic assessment of their job. Each hand diagram was scored independently by 2 raters.

Main Outcome Measures: Rating of the hand diagram for UN.

Results: Interrater reliability of scoring the hand diagram for UN was very high. The estimated prevalence of UN was $3.6 \%$. Suspected UN was associated with positioning of the elbow but not by contact stress at the elbow or force at the hand. Smokers had a lower prevalence, but smokers with suspected UN had higher-pack year histories. Workers with suspected UN had a greater sense of job insecurity and lower job satisfaction rating.

Conclusions: Hand diagram rating has a high interrater reliability. Suspected UN has a relatively high prevalence among active workers in comparison to prior estimates of the prevalence of UN among the general population and is not strongly associated with ergonomic factors.
\end{abstract}

\section{Introduction}

Ulnar neuropathy (UN) is a common nerve entrapment disorder in the upper extremity. Typical symptoms include numbness, tingling or burning sensation in the fourth and fifth digits of the hand. The most common site of entrapment is the elbow, although entrapment at the wrist is also a possibility. Although UN at the elbow is the second most common compression neuropathy of peripheral nerves, its prevalence in the workplace is not well defined. UN at the elbow has an annual incidence in the general population that is approximately 1 in 13 of that of carpal tunnel syndrome (CTS), or 24.7 per 100,000 population [1]. Another study estimated the prevalence rate as $0.6 \%$ to $0.8 \%$ [2]. There is little literature regarding the incidence or prevalence of this disorder in the working population, although 1 study of female floor cleaners demonstrated a prevalence of $7 \%$ [3].
There are no general population estimates of the prevalence of UN at the wrist.

Repetitive work was considered a risk factor in some studies but depended upon the case definition of UN $[4,5]$. UN has also been associated with forceful work and use of a tool in the hand $[5,6]$. UN at the elbow has been demonstrated to be more common in men and among smokers [7-10]. Sustained pressure over the elbow region is also considered a risk factor.

The diagnosis of UN at the elbow or Guyon canal nerve entrapment is typically made by clinical presentation and confirmed by electrodiagnostic testing. Clinically, an affected patient complains of numbness and tingling in an ulnar distribution. In a recent study, Werner et al [11] demonstrated that a hand diagram that shows numbness, tingling, burning, or pain in the ulnar distribution had a $50 \%$ sensitivity and $93 \%$ specificity with a receiver operating characteristic (ROC) curve of $90 \%$ when compared to electrodiagnostic 
testing of the ulnar nerve across the elbow. This demonstrated that the rating of a hand diagram for ulnar symptoms can be a valuable tool for estimating the prevalence of UN in the general population and in the workplace. This would also allow evaluation of associated ergonomic risk factors in a cost-effective manner. Hand diagrams have been used reliably for evaluation of CTS $[12,13]$.

It is known that there are other neurologic lesions that can present with a similar sensory distribution and thus a similar hand diagram. A C8 radiculopathy, lower trunk brachial plexopathy, or thoracic outlet syndrome can also present with symptoms in the fourth and fifth digits of the involved hand. The Werner et al study did not find any of these other etiologies in their screening of 117 patients [11]. The most common other neurologic abnormality was CTS or no abnormality at all on nerve conduction testing. Based upon the earlier Werner et al study [11], it is reasonable to assume that half of the subjects who screen positive for an abnormal ulnar hand diagram have UN, and this can be used to estimate the prevalence in the workplace.

\section{Methods}

A total of 501 active workers from 7 settings (4 industrial and 3 clerical work sites in southern Michigan and northern Ohio) were recruited for evaluation. This was part of a larger study previously described by Werner et al [14]. All subjects signed a consent form that was approved by the institutional review board at the University of Michigan. All subjects completed a symptom questionnaire, including a hand diagram. The hand diagram was rated for the possibility of an ulnar mononeuropathy using the classification protocol proposed by Werner et al [11] that is described in Table 1. The hand diagram was reviewed independently by 2 of the authors (A.F. and R.A.W.). For the interrater reliability study, all 3 categories of the hand diagram rating were used in the analysis. For the estimate of the prevalence of UN, a participant was considered to have a positive hand

Table 1

Hand Diagram Protocol for Screening for Ulnar Neuropathy

2 Definite Palmar symptoms in the fifth digit or symptoms in the fifth digit and ulnar side of hand. Symptoms may also be in the fourth digit, but cannot include digits 1,2 , or 3 or the radial aspect of the palm.

1 Possible Symptoms in the fifth digit and ulnar side hand. Symptoms may also be noted in the rest of the palm. Symptoms may be in digits: 1245,134 5 , or 1235 .

0 Unlikely No evidence of ulnar neuropathy-no symptoms in the fourth or fifth digit; or whole hand symptoms; or 4 contiguous digits involved; or only joint or nondistal phalanx involvement. diagram if either the right or left hand (or both) was rated as 1 (possible) or 2 (definite).

All subjects were weighed, and height was also recorded and used to determine their body mass index $\left(\mathrm{BMl} ; \mathrm{kg} / \mathrm{m}^{2}\right)$. All jobs were assessed and rated for ergonomic exposures at baseline and whenever a job change took place. The methods used to assess the jobs were described previously by Latko et al [15]. Jobs were videotaped and rated for the degree of repetition, average and peak hand contact stress, average and peak force, and average and peak posture of the shoulder, elbow, forearm, and wrist/hand. The ratings were performed using a 0-10 visual analog scale for each stressor, with verbal anchors on the $10-\mathrm{cm}$ scale. A rating of 0 corresponded to no stress (or neutral posture), and a rating of 10 corresponded to the greatest possible stress (or deviation from neutral). A neutral position for the elbow was defined as a $90^{\circ}$ angle. The formal ratings were conducted by a team comprising university faculty and research staff specializing in ergonomic analysis. The 3 team members rated the jobs independently, and then final ratings for the jobs were achieved through consensus. The same ergonomic risk factor measurement techniques have been adopted by the American Conference of Governmental Industrial Hygienists' (ACGIH) Threshold Limit Values (TLV) for evaluating hand activity level. The ratings for hand repetition level and the normalized peak force are multiplied and rated $(1=$ acceptable, 2 = borderline, 3 = unacceptable) [16]. The workers also completed a psychosocial questionnaire evaluating job security and satisfaction, supervisor and co-worker support, job creativity, decision authority, and skill discretion as defined by Karasek et al [17].

Statistical analysis included a $t$-test and $\chi^{2}$ test to determine which demographic, ergonomic, and psychosocial factors were associated with a higher prevalence of UN. Odds ratios were estimated using logistic regression modeling, with presence of a positive hand diagram as the dependent variable and the ergonomic, demographic, and psychosocial variables as the independent variables. A $\kappa$ value was determined to evaluate the interrater reliability.

\section{Results}

A total of 501 participants were recruited into the study. The mean age was $44.1 \pm 9.8$ years, with a range of $24-75$ years. Of the participants, $71 \%$ were female. The mean height was $1.67 \mathrm{~m}$, mean weight was $82.8 \mathrm{~kg}$, and mean BMI was $29.5 \mathrm{~kg} / \mathrm{m}^{2}$. A total of 36 subjects (7.2\%) had a classification of a possible or definite UN using the hand diagram criteria. Using the $50 \%$ sensitivity of a positive hand diagram for predicting UN, the estimated prevalence of suspected UN in this population was $3.6 \%$. The demographic, ergonomic, and psychosocial risk factors are presented in Table 2, 
Table 2

Demographic, ergonomic, and psychosocial risk factors stratified by positive or negative ulnar neuropathy hand diagram

\begin{tabular}{|c|c|c|c|}
\hline Characteristic & + Hand Diagram & - Hand Diagram & $P$ Value \\
\hline Age $(y)$ & $44.0(0.5)$ & $45.1(1.6)$ & .52 \\
\hline Gender (\% female) & 72.2 & 70.5 & .83 \\
\hline Weight $(\mathrm{kg})$ & $81.1(2.8)$ & $82.9(1.0)$ & .61 \\
\hline Height $(\mathrm{cm})$ & $1.67(0.02)$ & $1.67(0.01)$ & .05 \\
\hline $\mathrm{BMI} \mathrm{kg} / \mathrm{m}^{2}$ & $28.8(0.7)$ & $29.6(0.3)$ & .54 \\
\hline Hand repetition [1 (low) to 10 (high)] & $5.5(0.3)$ & $5.6(0.1)$ & .56 \\
\hline Hand TLV (\% unacceptable) & 17.1 & 27.5 & .41 \\
\hline Elbow positioning (average) [1 (low) to 10 (high)] & $3.1(0.2)$ & $3.4(0.04)$ & .08 \\
\hline Elbow positioning (peak) [1 (low) to 10 (high)] & $4.5(0.1)$ & $4.9(0.1)$ & .05 \\
\hline Elbow contact stress (average) [1 (low) to 10 (high)] & $0.01(0.002)$ & $0.02(0.003)$ & .32 \\
\hline Elbow contact stress (Peak) [1 (low) to 10 (high)] & $0.26(0.02)$ & $0.31(0.02)$ & .52 \\
\hline Job dissatisfaction* & $0.36(0.03)$ & $0.30(0.01)$ & .07 \\
\hline Supervisor support ${ }^{*}$ & $10.7(0.5)$ & $11.3(1)$ & .18 \\
\hline Co-worker support* & $11.2(0.4)$ & $11.8(0.1)$ & .07 \\
\hline Job insecurity* & $5.1(0.4)$ & $4.4(0.1)$ & .04 \\
\hline Decision authority* & $24.7(1.7)$ & $26.2(0.4)$ & .34 \\
\hline Skill discretion* & $24.8(1.6)$ & $25.5(0.4)$ & .61 \\
\hline Smoking history (\% smokers) & 25 & 41 & .05 \\
\hline Smoking pack-years & $25.8(3.7)$ & $20.3(0.8)$ & .08 \\
\hline
\end{tabular}

Data are mean (standard error).

$+=$ Positive; $-=$ negative; $\mathrm{BMI}=$ body mass index; TLV $=$ threshold limit value

* From the Job Content Questionnaire [17].

stratified by workers with and without a positive hand diagram for UN.

Age, height, weight, and BMI were not associated with a positive hand diagram. Hand repetition, elbow contact stress and hand TLV also were not associated with a positive hand diagram. Less variability in elbow positions during the work day was related to a higher prevalence of suspected UN. Workers with suspected UN were less likely to smoke but had a trend for higher total pack-years for those who did smoke. Psychosocial variables defined by Karasek et al [17] did have some influence on suspected UN. Workers with suspected UN had lower job satisfaction and co-worker support as well as higher job insecurity. Other measures such as skill discretion, decision authority, and supervisor support did not influence the prevalence of suspected UN.

Backward stepwise logistic regression analysis demonstrated a model that included elbow position, job dissatisfaction, job insecurity, and hand repetition as the significant variables using $P<.2$ as a cutoff for dropping independent variables. The model had a low pseudo- $R^{2}$ of 0.09 . The odds ratios for this model are shown in Table 3.

Table 3

Logistic regression model (backward stepwise) using the ulnar hand diagram as the dependent variable and the demographic, ergonomic, and job content variables as the independent variable

\begin{tabular}{lcl}
\hline Variable & Odds Ratio & 95\% Confidence Interval \\
\hline Elbow position & 0.31 & $0.11,0.84$ \\
Job dissatisfaction & 13.0 & $1.0,163.0$ \\
Job insecurity & 1.29 & $1.00,1.65$ \\
Hand repetition & 1.38 & $0.88,2.15$ \\
\hline
\end{tabular}

$P<.2$ was used as the exclusionary level. Pseudo $R^{2}=0.09$.
In the interrater reliability analysis, the $\kappa$ score was 0.93 when all hand diagrams were included. Because many subjects in this cohort had no symptoms in their hands, we also analyzed the data with only those subjects who had some symptoms in the ulnar distribution. In this subset, the $\kappa$ value was 0.82 .

\section{Discussion}

The study by Werner et al [11] demonstrates that the ulnar hand diagram criteria have a fair to good sensitivity for identifying true cases of UN and high specificity. We were able to show a very high interrater reliability when grading the hand diagram. Prevalence of suspected UN in this population was estimated by using the $50 \%$ sensitivity for the hand diagram with the grading system described in Table 1 . The estimated prevalence was $3.6 \%$, which is relatively high in comparison to prior estimates of the prevalence of UN in the general population, and is similar to estimates of the prevalence of CTS in general population [18]. It is less than the $7 \%$ among female floor washers described by Mondelli et al [3]. Floor washing would be considered a relative high-risk occupation for UN.

There were few demographic or ergonomic risk factors identified in this cross-sectional sample of active workers from multiple industrial and clerical sites. The only ergonomic factors identified were related to elbow posture, and, surprisingly, neutral elbow postures were associated with a higher risk of suspected UN. The difference was statistically significant, but the measurable difference was small and may not be helpful in the individual assessment of a job. This finding is contrary to the anticipated relationship of greater elbow flexion and 
extension being a mechanical factor that may predispose individuals to the development of UN. The ergonomic metric was relative deviation of the elbow joint from a $90^{\circ}$ position. It was not a measure of number of repetitive elbow movements, which may be a more critical aspect of risk for UN. This is a limitation of the ergonomic assessment tool used, and future studies might consider measuring elbow flexion/extension repetition.

Smoking history was lower among workers with suspected UN, which is also contrary to other epidemiologic studies that have found a higher prevalence of smoking among patients with UN [7-10]. One consideration is that in today's smoke-free work environment, taking a smoking break away from the work station may allow recovery time for a possible compression neuropathy. On the other hand, if the worker with suspected UN did smoke, that individual had a higher pack-year history compared to workers without UN who smoked.

Lower job satisfaction, lower co-worker support, and higher job insecurity were all associated with a higher prevalence of suspected UN. These psychosocial variables may have influenced more workers to report symptoms when filling out the symptom questionnaire. This relationship is common with workers with back pain but has not been demonstrated among workers with UN.

The limitations of the study include use of a hand diagram to estimate UN as opposed to electrodiagnostic criteria for UN. The use of the hand diagram has been demonstrated to have a sensitivity of $50 \%$ and a specificity of $93 \%$, but it is still an estimate. The sample size was modest at 501, with only 36 workers identified with a positive ulnar hand diagram. A larger sample size would provide a better estimate of the prevalence. In addition, the sample was more than $70 \%$ female and included a high percentage of smokers, which limits the generalizability to the entire population of active workers. The analysis of risk factors is based upon the identification of an abnormal hand diagram; these are not the same as confirmed cases of UN, and therefore the findings may be confounded by the inclusion of workers with other disorders. Replication of these findings would be important to understanding the true relationship among ergonomic factors, psychosocial factors, and abnormal hand diagrams.

\section{Conclusion}

Hand diagram rating for UN was found to have a high interrater reliability. Suspected UN had a relatively high prevalence among active workers in comparison to prior estimates of the prevalence of UN among the general population, and is not strongly associated with ergonomic factors

\section{Acknowledgments}

Support for this research is provided by the National Institute on Disability and Rehabilitation Research of the United States Department of Education, grant \#H133E980007, "Rehabilitation Engineering Research Center." The opinions contained in this publication are those of the grantee and do not necessarily reflect those of the United States Department of Education.

\section{References}

1. Mondelli M, Giannini F, Ballerini M, et al. Incidence of ulnar neuropathy at the elbow in the province of Siena (Italy). J Neurol Sci 2005;15:5-10.

2. Roquelaure $\mathrm{Y}, \mathrm{Ha}$ C, Leclerc A, et al. Epidemiologic surveillance of upper-extremity musculoskeletal disorders in the working population. Arthritis Rheum 2006;55:765-778.

3. Mondelli M, Grippo A, Mariani M, et al. Carpal tunnel syndrome and ulnar neuropathy at the elbow in floor cleaners. Neurophysiol Clin 2006;36:245-253.

4. Descatha A, Leclerc A, Chastang JF, et al. Study Group on Repetitive-Work. Incidence of ulnar nerve entrapment at the elbow in repetitive work. Scand J Work Environ Health 2004;30: 234-240.

5. Svendsen SW, Johnsen B, Fuglsang-Frederiksen A, Frost P. Ulnar neuropathy and ulnar neuropathy-like symptoms in relation to biomechanical exposures assessed by a job exposure matrix: A triple case-referent study. Occup Environ Med 2012;69:773-780.

6. van Rijn R, Huisstede B, Koes B, Burdorf A. Associations between work-related factors and specific disorders at the elbow: A systematic literature review. Rheumatology 2009;48:528-536.

7. Bartels RH, Verbeek AL. Risk factors for ulnar nerve compression at the elbow: A case control study. Acta Neurochirurgic 2007;149: 669-674.

8. Richardson JK, Green DF, Jamieson SC, Valentin FC. Gender, body mass and age as risk factors for ulnar mononeuropathy at the elbow. Muscle Nerve 2001;24:551-554.

9. Richardson JK, Jamieson SC. Cigarette smoking and ulnar mononeuropathy at the elbow. Am J Phys Med Rehabil 2004;83:730-734.

10. Frost P, Johnsen B, Fuglsang-Frederiksen A, Svendsen S. Lifestyle risk factors for ulnar neuropathy and ulnar neuropathy-like symptoms. Muscle Nerve 2013;48:507-515.

11. Werner RA, Chiodo A, Spiegelberg T, Franzblau A. Use of hand diagrams for screening for ulnar neuropathy; comparison with electrodiagnostic studies. Muscle Nerve 2012;46:891-894.

12. Katz JN, Stirrat CR. A self-administered hand diagram for the diagnosis of carpal tunnel syndrome. J Hand Surg 1990;15A: 360-363.

13. Katz JN, Stirrat CR, Larson MG, Fossel AH, Eaton HM, Liang MH. A self-administered hand symptom diagram for the diagnosis and epidemiologic study of carpal tunnel syndrome. J Rheumatol 1990; 17:1495-1498.

14. Werner RA, Gell N, Franzblau A, Ulin SS, Armstrong TJ. Predictors of upper extremity discomfort: A longitudinal study of industrial and clerical workers. J Occup Rehabil 2005;15:27-35.

15. Latko WA, Armstrong TJ, Foulke JA, Herrin GD, Rabourn RA, Ulin SS. Development and evaluation of an observational method for assessing repetition in hand tasks. Am Indust Hyg Assoc J 1997; 58:278-285.

16. 2002 Threshold Limit Values for Chemical Substances and Physical Agents in the Work Environment. Cincinnati, OH: ACGIH Worldwide, 2002.

17. Karasek R, Brisson C, Kawakami N, Houtman I, Bongers P, Amick B. The Job Content Questionnaire (JCQ): An instrument for internationally comparative assessments of psychosocial job characteristics. J Occup Health Psychol 1998;3:322-355.

18. Atroshi I, Gummesson C, Johnsson R, Ornstein E, Ranstam J, Rosen I. Prevalence of carpal tunnel syndrome in a general population. JAMA 1999;282:153-158. 
This journal-based CME activity is designated for 1.0 AMA PRA Category 1 Credit $^{\mathrm{TM}}$ and can be completed online at wWw.me.aapmr.org. This activity is FREE to AAPM\&R members and available to nonmembers for a nominal fee. For assistance with claiming CME for this activity, please contact (847) 737-6000.

\section{Disclosure}

R.A.W. Department of Physical Medicine and Rehabilitation, Veterans Administration Health System, 2215 Fuller Rd. (117), Ann Arbor, MI 48105; Department of Physical Medicine and Rehabilitation, University of Michigan Health Systems, Ann Arbor, MI; Department of Environmental Health Sciences, School of Public Health, University of Michigan, Ann Arbor, MI; Center for Ergonomics, College of Engineering, University of Michigan, Ann Arbor, MI. Address correspondence to: R.A.W.; e-mail: rawerner@umich.edu

Disclosures related to this publication: grant, National Institute on Disability and Rehabilitation Research (NIDRR) (money to institution)

A.F. Department of Environmental Health Sciences, School of Public Health, University of Michigan, Ann Arbor, MI

Disclosure: nothing to disclose
S.U. Center for Ergonomics, College of Engineering, University of Michigan, Ann Arbor, MI

Disclosures related to this publication: NIDRR, grant \#H133E980007 (money to institution)

A poster of these findings was presented at the national meeting of the American Association of Neuromuscular and Electrodiagnostic Medicine in Savannah, GA, in October 2014.

Peer reviewers and all others who control content have no relevant financial relationships to disclose.

Submitted for publication September 10, 2014; accepted December 21, 2014.

B.E. Department of Public Health, Washington University, St. Louis, MO Disclosure: nothing to disclose

\section{CME Question}

The most common location for ulnar nerve compression is?
a. Guyon canal
b. arcade of Struthers
c. flexor carpi ulnaris aponeurosis
d. anterior scalene

Answer online at me.aapmr.org 